
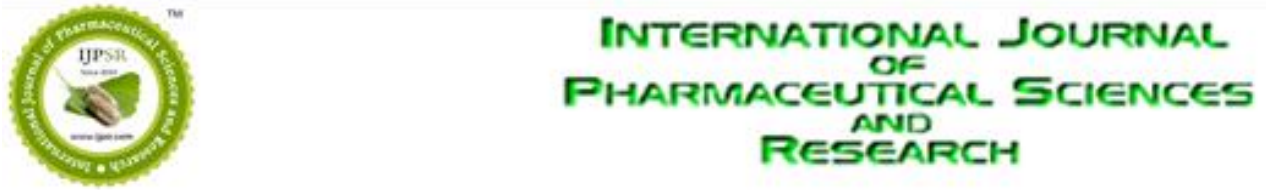

Received on 29 April, 2013; received in revised form, 15 July, 2013; accepted, 21 August, 2013; published 01 September, 2013

\title{
COMPARATIVE STUDY OF OVERLAPPING GENES IN THE GENOMES OF MYCOPLASMA HOMINIS AND MYCOPLASMA PENETRANS
}

Kandavelmani Angamuthu* ${ }^{1}$ and Shanmughavel Piramanayagam ${ }^{2}$

DBT Bioinformatics Facility, Department of Bioinformatics ${ }^{1}$, Research and Development Centre ${ }^{2}$, Bharathiar University, Coimbatore - 641046, Tamil Nadu, India

Keywords:

Obligatory parasites, Frame shift, Evolution

Correspondence to Author:

Kandavelmani Angamuthu

Research and Development Centre, DBT Bioinformatics Facility,

Department of Bioinformatics,

Bharathiar University, Coimbatore -

641046, Tamil Nadu, India

E-mail: kandavelmani@gmail.com
ABSTRACT: Overlapping genes are pairs of adjacent genes whose coding regions are partially or entirely overlapping. Overlapping genes in prokaryotes are a means to minimize genome size and increase the density of genetic information. Overlapping genes play an important role in genome reduction of Mycoplasmas during the course of their degenerative evolution. In this study overlapping genes in the genomes of two obligatory human parasites, Mycoplasma hominis and Mycoplasma penetrans were extracted and systematically analyzed. Overlapping genes were classified into different categories based on their direction of transcription. Gene pairs that occur as overlapping in both the genomes, gene pairs that occur as overlapping in one genome but are split in the other genome were separated out and more closely analysed. The study revealed that most of the overlapping genes in these genomes are formed due to loss of a stop codon or frame shift. The present study also emphasizes the significance of overlapping genes in the evolution of these genomes.
INTRODUCTION: Overlapping genes are pairs of adjacent genes whose coding regions are partially or entirely overlapping. Overlapping genes are a common occurrence in viruses, bacteria and mitochondria.

Overlapping genes have also been identified in various eukaryotic organisms including humans and systematic analysis methods have been developed ${ }^{1,2}$. Overlapping genes have evolved due to mutational bias towards deletion ${ }^{3}$.

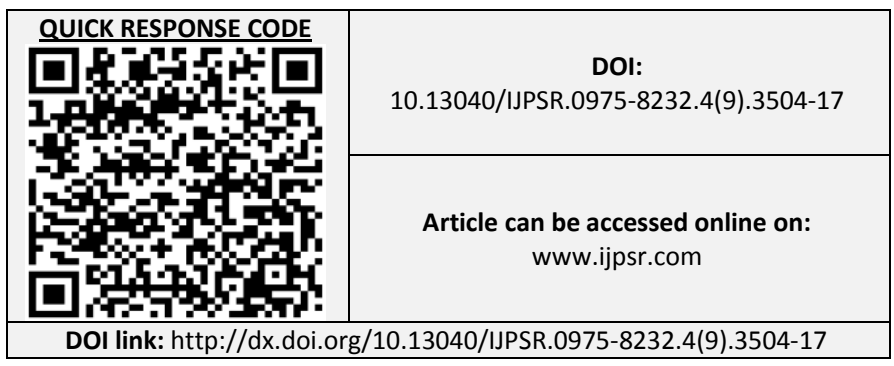

Evidence from previous studies show that most overlapping genes have originated as a result of stop codon deletion, by a point mutation at the stop codon or by the introduction of a near - end frameshift extending the protein translation till the next in-frame stop codon ${ }^{4-6}$.

Overlapping genes are also found to evolve due to the acquisition of an upstream start codon by the downstream gene ${ }^{7}$. Genomic overlaps in prokaryotes may be a result of evolutionary pressure to minimize genome size and increase the density of genetic information ${ }^{8}$.

Overlapping genes have been suggested to have multiple functions such as regulation of gene expression, translational coupling and genome overprinting - a process of generating novel genes through accumulated mutations inside a pre existing gene ${ }^{9,10}$. 
Overlapping genes are more conserved between species than non-overlapping genes mostly because a mutation in the overlapping region causes changes in both genes ${ }^{11,12}$. Overlapping genes can be used as rare genomic markers to get insight into the phylogeny of the completely sequenced microbial genomes ${ }^{13}$. Mycoplasmas, the obligatory parasites of humans, animals and plants seem to have evolved more rapidly than other bacteria since some highly variable positions in their rRNA sequences are strongly conserved in other bacterial species ${ }^{14}$.

Mycoplasmas have evolved from Gram-positive bacteria by reductive evolution thereby losing several genes involved in metabolism, cellular process and energy production 15, 16. However, overlapping genes have been proposed as means of achieving genome reduction by retaining indispensable genes and compressing maximum amount of information in available sequence space 17. As an endeavor to analyze the role of overlapping genes in genome reduction, in the present study all the overlapping genes in the genomes of the two obligatory human parasites, Mycoplasma hominis and Mycoplasma penetrans were systematically analyzed.

MATERIALS AND METHODS: The genome sequences of two obligatory human parasites, $M$. hominis and $M$. penetrans were downloaded from the National Center for Biotechnology Information website (ftp://ftp.ncbi.nih.gov/genomes/Bacteria/).

Overlapping genes, defined as pairs of adjacent genes whose coding regions partly or completely overlap were extracted using the (CDS) annotation feature. The extracted overlapping gene pairs were classified into three directional patterns, namely, 'convergent' $(\rightarrow \leftarrow)$, 'unidirectional' $(\rightarrow \longrightarrow, \leftarrow \leftarrow)$, and 'divergent' $(\longleftrightarrow \longrightarrow)$ based on their direction of transcription ${ }^{18}$. The overlapping gene pairs of M.penetrans and M.hominis were further classified into four categories, (i) Gene pairs that occur as overlapping in both genomes

(ii) Gene pairs that overlap in both genomes with different number of overlapping bases

(iii)Gene pair that overlap in $M$. penetrans but are split in M. hominis

(iv) Gene pairs that overlap in $M$. hominis but are split in $M$. penetrans.

The gene name, function, direction of overlap and the number of overlapping bases were tabulated for each category an analysed ${ }^{6}$.

RESULTS AND DISCUSSION: The number overlapping genes and their orientation in the genomes of $M$. hominis and $M$. penetrans are summarized in table 1. Most of the overlapping genes are unidirectional, a few pairs are convergent and only one gene pair in $M$. hominis is divergent. These results concur with the earlier hypothesis that most overlapping gene pairs have unidirectional structure ${ }^{19}$. This is a common occurrence in prokaryote genomes because in prokaryotes adjacent genes with the same orientation are often organized into operons or clusters and are transcribed together in the same direction.

It has been previously reported that the number of overlapping genes increases with the genome size and the number of ORFs ${ }^{5}$. In contrast to this notion, in the current study it is observed that $M$. penetrans with a comparatively larger genome size and higher number of genes is found to have lesser number of overlapping gene pairs than that of $M$. hominis (Table 1). It has been reported earlier that Mycoplasma genitalium with the smallest genome has the largest proportion of overlapping gene pairs 6 . This clearly proves that overlapping genes play an important role in genome compaction of the minimal genomes of Mycoplasmas during their course of degenerative evolution.

TABLE 1: NUMBER OF OVERLAPPING GENE PAIRS AND THEIR ORIENTATION

\begin{tabular}{|c|c|c|c|c|c|c|c|c|}
\hline & \multirow{3}{*}{$\begin{array}{c}\text { Genome } \\
\text { Size }(\mathrm{Mb})\end{array}$} & \multirow{3}{*}{$\begin{array}{l}\text { No. of } \\
\text { Genes }\end{array}$} & \multirow{3}{*}{$\begin{array}{c}\text { No. of } \\
\text { Overlapping } \\
\text { gene pairs }\end{array}$} & \multirow{3}{*}{$\begin{array}{c}\% \text { of } \\
\text { Overlapping } \\
\text { genes }\end{array}$} & \multicolumn{4}{|c|}{ Orientation of overlapping gene pairs } \\
\hline & & & & & \multicolumn{2}{|c|}{ Unidirectional } & \multirow{2}{*}{$\begin{array}{c}\text { Convergent } \\
\rightarrow \leftarrow \\
\end{array}$} & \multirow{2}{*}{$\begin{array}{c}\text { Divergent } \\
\leftarrow \rightarrow\end{array}$} \\
\hline & & & & & $\leftarrow \leftarrow$ & $\rightarrow \rightarrow$ & & \\
\hline M. hominis & 0.67 & 577 & 111 & 19.2 & 45 & 61 & 4 & 1 \\
\hline M. penetrans & 1.36 & 1069 & 109 & 10.1 & 52 & 51 & 6 & 0 \\
\hline
\end{tabular}


Overlapping genes, number of overlapping nucleotides (length) and their direction of overlap in the genomes of M. hominis and M. penetrans are listed in table $2 \& 3$ respectively. Among all the unidirectional overlapping gene pairs 35 pairs in $M$. hominis and 15 pairs $M$. penetrans overlap only by one base. Most of the overlapping genes in $M$. hominis and M. penetrans are formed due to loss of a stop codon or frame shift.

Table 4 summarizes the gene pairs that are found to be overlapping in both the genomes. All the 12 common overlapping gene pairs are found to have unidirectional orientation.

Among the 12 common overlapping gene pairs, five gene pairs overlap by the same number of nucleotides.
Whereas, seven other common overlaps differ in their overlapping lengths. The five common unidirectional overlapping gene pairs are found on the same strand in both the genomes except for MHO_1230: MHO_1240 $(\rightarrow \rightarrow)$ and MYPE7420: MYPE7430 $(\leftarrow \leftarrow)$. There are 36 gene pairs that are overlapping in $M$. hominis but are nonoverlapping in $M$. penetrans (Table 5). Whereas on the other hand, there are 17 gene pairs that are overlapping in $M$. penetrans but are split in $M$. hominis which has a smaller genome (Table 6). These discrepancies are in accordance with the notion that overlapping genes may be a means of compressing maximum amount of information into the available short sequence space may be a result of evolutionary pressure to minimize the genome size and increase the density of genetic information 20

TABLE 2: OVERLAPPING GENES IN M. HOMINIS

\begin{tabular}{ccccc}
\hline S. No. & Genes & Genes & Length & Direction \\
\hline 1 & MHO_0010 & rnpA & 26 & $\leftarrow \leftarrow$ \\
2 & dnaN & MHO_0060 & 1 & $\rightarrow \rightarrow$ \\
3 & ksgA & MHO_0090 & 8 & $\leftarrow \leftarrow$ \\
4 & MHO_0090 & MHO_0100 & 1 & $\leftarrow \leftarrow$ \\
5 & MHO_0190 & atpB & 8 & $\rightarrow \rightarrow$ \\
6 & atpA & atpG & 22 & $\rightarrow \rightarrow$ \\
7 & atpD & atpC & 1 & $\rightarrow \rightarrow$ \\
8 & scpA & scpB & 11 & $\rightarrow \rightarrow$ \\
9 & scpB & rluC & 14 & $\rightarrow \rightarrow$ \\
10 & gatC & gatA & 11 & $\rightarrow \rightarrow$ \\
11 & gatA & gatB & 8 & $\rightarrow \rightarrow$ \\
12 & gatB & MHO_0460 & 8 & $\rightarrow \rightarrow$ \\
13 & MHO_0580 & MHO_0590 & 1 & $\rightarrow \rightarrow$ \\
14 & cysS & MHO_0620 & 1 & $\rightarrow \rightarrow$ \\
15 & MHO_0660 & fba & 17 & $\leftarrow$ \\
16 & MHO_0740 & MHO_0750 & 1 & $\rightarrow \rightarrow$ \\
17 & MHO_0750 & MHO_0760 & 59 & $\rightarrow \rightarrow$ \\
18 & MHO_0840 & uvrC & 11 & $\rightarrow$ \\
19 & rplk & rplA & 1 & $\rightarrow$ \\
\hline
\end{tabular}


Angamuthu and Piramanayagam, IJPSR, 2013; Vol. 4(9): 3504-3517. $\quad$ E-ISSN: 0975-8232; P-ISSN: 2320-5148

\begin{tabular}{|c|c|c|c|c|}
\hline 20 & MHO_0960 & recR & 1 & $\rightarrow \rightarrow$ \\
\hline 21 & dnaH & MHO_1000 & 8 & $\rightarrow \rightarrow$ \\
\hline 22 & thrS & $\operatorname{trpS}$ & 1 & $\leftarrow \leftarrow$ \\
\hline 23 & MHO_1070 & MHO_1080 & 8 & $\rightarrow \rightarrow$ \\
\hline 24 & MHO_1080 & MHO_1090 & 50 & $\rightarrow \rightarrow$ \\
\hline 25 & nusB & tlyA & 17 & $\rightarrow \rightarrow$ \\
\hline 26 & tlyA & nifS & 1 & $\rightarrow \rightarrow$ \\
\hline 27 & nifS & nifU & 14 & $\rightarrow \rightarrow$ \\
\hline 28 & nifU & mucB & 8 & $\rightarrow \rightarrow$ \\
\hline 29 & mucB & MHO_1260 & 1 & $\rightarrow \rightarrow$ \\
\hline 30 & alaS & MHO_1320 & 13 & $\rightarrow \rightarrow$ \\
\hline 31 & MHO_1320 & MHO_1330 & 8 & $\rightarrow \rightarrow$ \\
\hline 32 & MHO_1330 & MHO_1340 & 11 & $\rightarrow \rightarrow$ \\
\hline 33 & MHO_1340 & greA & 1 & $\rightarrow \rightarrow$ \\
\hline 34 & MHO_1400 & rluD & 2 & $\leftarrow \rightarrow$ \\
\hline 35 & oppD & oppF & 8 & $\rightarrow \rightarrow$ \\
\hline 36 & MHO_1570 & MHO_1580 & 1 & $\rightarrow \rightarrow$ \\
\hline 37 & prfA & hemK & 1 & $\rightarrow \rightarrow$ \\
\hline 38 & $\mathrm{cmk}$ & eng A & 14 & $\rightarrow \rightarrow$ \\
\hline 39 & MHO_1710 & MHO_1720 & 14 & $\leftarrow \leftarrow$ \\
\hline 40 & oppB & oppC & 17 & $\rightarrow \rightarrow$ \\
\hline 41 & tktA & MHO_1780 & 11 & $\leftarrow \leftarrow$ \\
\hline 42 & ruvA & ruvB & 26 & $\rightarrow \rightarrow$ \\
\hline 43 & hisS & aspS & 17 & $\rightarrow \rightarrow$ \\
\hline 44 & dgk & hpt & 13 & $\rightarrow \rightarrow$ \\
\hline 45 & cbiO1 & cbiO2 & 10 & $\rightarrow \rightarrow$ \\
\hline 46 & cbiO2 & cbiQ & 8 & $\rightarrow \rightarrow$ \\
\hline 47 & $\operatorname{lig} \mathrm{A}$ & MHO_2010 & 24 & $\rightarrow \leftarrow$ \\
\hline 48 & MHO_2010 & MHO_2020 & 76 & $\leftarrow \leftarrow$ \\
\hline 49 & MHO_2060 & pgsA & 11 & $\rightarrow \rightarrow$ \\
\hline 50 & smf & nfo & 9 & $\rightarrow \leftarrow$ \\
\hline 51 & MHO_2190 & MHO_2200 & 8 & $\leftarrow \leftarrow$ \\
\hline
\end{tabular}


Angamuthu and Piramanayagam, IJPSR, 2013; Vol. 4(9): 3504-3517. $\quad$ E-ISSN: 0975-8232; P-ISSN: 2320-5148

\begin{tabular}{|c|c|c|c|c|}
\hline 52 & MHO_2330 & MHO_2340 & 20 & $\leftarrow \leftarrow$ \\
\hline 53 & MHO_2370 & ribF & 1 & $\rightarrow \rightarrow$ \\
\hline 54 & MHO_2450 & MHO_2460 & 1 & $\leftarrow \leftarrow$ \\
\hline 55 & infA & map & 1 & $\leftarrow \leftarrow$ \\
\hline 56 & map & adk & 10 & $\leftarrow \leftarrow$ \\
\hline 57 & adk & $\sec \mathrm{Y}$ & 7 & $\leftarrow \leftarrow$ \\
\hline 58 & $\sec Y$ & $\mathrm{rplO}$ & 1 & $\leftarrow \leftarrow$ \\
\hline 59 & $\mathrm{rpsE}$ & rplR & 1 & $\leftarrow \leftarrow$ \\
\hline 60 & rplN & rpsQ & 8 & $\leftarrow \leftarrow$ \\
\hline 61 & rpsQ & $\mathrm{rpmC}$ & 1 & $\leftarrow \leftarrow$ \\
\hline 62 & $\mathrm{rplP}$ & $\mathrm{rpsC}$ & 23 & $\leftarrow \leftarrow$ \\
\hline 63 & $\mathrm{rpsC}$ & $\mathrm{rplV}$ & 1 & $\leftarrow \leftarrow$ \\
\hline 64 & $\mathrm{rpsS}$ & rplB & 1 & $\leftarrow \leftarrow$ \\
\hline 65 & rplW & rplD & 1 & $\leftarrow \leftarrow$ \\
\hline 66 & rplD & $\mathrm{rplC}$ & 1 & $\leftarrow \leftarrow$ \\
\hline 67 & $\operatorname{lsp} A$ & ileS & 11 & $\leftarrow \leftarrow$ \\
\hline 68 & MHO_3080 & MHO_3090 & 14 & $\rightarrow \rightarrow$ \\
\hline 69 & atpD & atpA & 1 & $\leftarrow \leftarrow$ \\
\hline 70 & MHO_3140 & MHO_3150 & 8 & $\leftarrow \leftarrow$ \\
\hline 71 & MHO_3150 & MHO_3160 & 23 & $\leftarrow \leftarrow$ \\
\hline 72 & MHO_3170 & MHO_3180 & 20 & $\leftarrow \leftarrow$ \\
\hline 73 & hsdS & hsdS & 23 & $\leftarrow \leftarrow$ \\
\hline 74 & MHO_3280 & MHO_3290 & 1 & $\leftarrow \leftarrow$ \\
\hline 75 & $\mathrm{rnhB}$ & MHO_3310 & 8 & $\leftarrow \leftarrow$ \\
\hline 76 & MHO_3360 & MHO_3370 & 14 & $\rightarrow \leftarrow$ \\
\hline 77 & uvrA & MHO_3430 & 11 & $\leftarrow \leftarrow$ \\
\hline 78 & MHO_3440 & MHO_3450 & 38 & $\leftarrow \leftarrow$ \\
\hline 79 & gidB & prs & 26 & $\leftarrow \leftarrow$ \\
\hline 80 & ldh & pgi & 8 & $\leftarrow \leftarrow$ \\
\hline 81 & pgi & rpsA & 11 & $\leftarrow \leftarrow$ \\
\hline 82 & pepF & apt & 1 & $\leftarrow \leftarrow$ \\
\hline 83 & md2 & md1 & 8 & $\leftarrow \leftarrow$ \\
\hline
\end{tabular}


Angamuthu and Piramanayagam, IJPSR, 2013; Vol. 4(9): 3504-3517. $\quad$ E-ISSN: 0975-8232; P-ISSN: 2320-5148

\begin{tabular}{|c|c|c|c|c|}
\hline 84 & rpII & MHO_3930 & 20 & $\leftarrow \leftarrow$ \\
\hline 85 & engB & gcp & 1 & $\leftarrow \leftarrow$ \\
\hline 86 & gcp & MHO_3960 & 7 & $\leftarrow \leftarrow$ \\
\hline 87 & MHO_3960 & MHO_3970 & 1 & $\leftarrow \leftarrow$ \\
\hline 88 & MHO_4000 & rpmG & 1 & $\leftarrow \leftarrow$ \\
\hline 89 & dnaE & polA & 14 & $\rightarrow \rightarrow$ \\
\hline 90 & recD & pth & 1 & $\rightarrow \rightarrow$ \\
\hline 91 & pth & tils & 14 & $\rightarrow \rightarrow$ \\
\hline 92 & MHO_4320 & ung & 11 & $\rightarrow \leftarrow$ \\
\hline 93 & MHO_4380 & gltX & 20 & $\leftarrow \leftarrow$ \\
\hline 94 & potA & potB & 32 & $\rightarrow \rightarrow$ \\
\hline 95 & potB & potC & 8 & $\rightarrow \rightarrow$ \\
\hline 96 & MHO_4500 & MHO_4510 & 7 & $\rightarrow \rightarrow$ \\
\hline 97 & pyrH & frr & 1 & $\rightarrow \rightarrow$ \\
\hline 98 & MHO_4660 & MHO_4670 & 1 & $\rightarrow \rightarrow$ \\
\hline 99 & plsX & rnc & 11 & $\rightarrow \rightarrow$ \\
\hline 100 & MHO_4750 & MHO_4760 & 10 & $\rightarrow \rightarrow$ \\
\hline 101 & MHO_4760 & MHO_4770 & 26 & $\rightarrow \rightarrow$ \\
\hline 102 & MHO_4870 & sps1 & 8 & $\rightarrow \rightarrow$ \\
\hline 103 & MHO_4950 & bcrA & 1 & $\rightarrow \rightarrow$ \\
\hline 104 & bcrA & MHO_4970 & 23 & $\rightarrow \rightarrow$ \\
\hline 105 & MHO_4970 & MHO_4980 & 8 & $\rightarrow \rightarrow$ \\
\hline 106 & MHO_4980 & MHO_4990 & 23 & $\rightarrow \rightarrow$ \\
\hline 107 & nusA & MHO_5260 & 7 & $\rightarrow \rightarrow$ \\
\hline 108 & MHO_5260 & $\operatorname{infB}$ & 49 & $\rightarrow \rightarrow$ \\
\hline 109 & $\operatorname{infB}$ & rbfA & 14 & $\rightarrow \rightarrow$ \\
\hline 110 & MHO_5310 & MHO_5320 & 8 & $\rightarrow \rightarrow$ \\
\hline 111 & MHO_5330 & MHO_5340 & 1 & $\leftarrow \leftarrow$ \\
\hline
\end{tabular}

TABLE 3: OVERLAPPING GENES IN M. PENETRANS

\begin{tabular}{ccccc}
\hline S. No. & Genes & Genes & Length & Direction \\
\hline $\mathbf{1}$ & spoU & MYPE240 & 10 & $\rightarrow \rightarrow$ \\
$\mathbf{2}$ & MYPE340 & MYPE350 & 32 & $\rightarrow \rightarrow$ \\
\hline
\end{tabular}


Angamuthu and Piramanayagam, IJPSR, 2013; Vol. 4(9): 3504-3517. $\quad$ E-ISSN: 0975-8232; P-ISSN: 2320-5148

\begin{tabular}{|c|c|c|c|c|}
\hline 3 & MYPE380 & scpB & 11 & $\rightarrow \rightarrow$ \\
\hline 4 & MYPE400 & engB & 1 & $\rightarrow \rightarrow$ \\
\hline 5 & MYPE430 & MYPE440 & 109 & $\rightarrow \rightarrow$ \\
\hline 6 & MYPE450 & MYPE460 & 23 & $\rightarrow \rightarrow$ \\
\hline 7 & MYPE490 & fmt & 40 & $\rightarrow \rightarrow$ \\
\hline 8 & $\operatorname{prfA}$ & hemK & 35 & $\rightarrow \rightarrow$ \\
\hline 9 & MYPE670 & MYPE680 & 35 & $\rightarrow \rightarrow$ \\
\hline 10 & hrcA & MYPE720 & 26 & $\rightarrow \rightarrow$ \\
\hline 11 & nusA & MYPE1100 & 8 & $\rightarrow \rightarrow$ \\
\hline 12 & $\operatorname{infB}$ & rbfA & 17 & $\rightarrow \rightarrow$ \\
\hline 13 & nfo & fur & 13 & $\rightarrow \rightarrow$ \\
\hline 14 & MYPE1280 & MYPE1290 & 17 & $\rightarrow \rightarrow$ \\
\hline 15 & MYPE1290 & MYPE1300 & 26 & $\rightarrow \leftarrow$ \\
\hline 16 & MYPE1300 & rluB & 11 & $\leftarrow \leftarrow$ \\
\hline 17 & Lsp & MYPE1330 & 8 & $\leftarrow \leftarrow$ \\
\hline 18 & MYPE1440 & pth & 8 & $\rightarrow \rightarrow$ \\
\hline 19 & MYPE1460 & MYPE1470 & 7 & $\rightarrow \rightarrow$ \\
\hline 20 & mucB & MYPE1500 & 11 & $\leftarrow \leftarrow$ \\
\hline 21 & Efp & MYPE1590 & 1 & $\rightarrow \rightarrow$ \\
\hline 22 & MYPE1590 & nusB & 11 & $\rightarrow \rightarrow$ \\
\hline 23 & MYPE1610 & MYPE1620 & 11 & $\rightarrow \rightarrow$ \\
\hline 24 & MYPE1620 & MYPE1630 & 38 & $\rightarrow \rightarrow$ \\
\hline 25 & Dam & dam & 38 & $\rightarrow \rightarrow$ \\
\hline 26 & MYPE1830 & $\operatorname{lig} \mathrm{A}$ & 23 & $\rightarrow \rightarrow$ \\
\hline 27 & MYPE1970 & dnaE & 23 & $\rightarrow \rightarrow$ \\
\hline 28 & Fpg & MYPE2010 & 28 & $\rightarrow \rightarrow$ \\
\hline 29 & MYPE2020 & MYPE2030 & 44 & $\rightarrow \rightarrow$ \\
\hline 30 & gat $\mathrm{A}$ & gatB & 23 & $\rightarrow \rightarrow$ \\
\hline 31 & MYPE2200 & MYPE2210 & 8 & $\leftarrow \leftarrow$ \\
\hline 32 & eng $A$ & gpsA & 11 & $\rightarrow \rightarrow$ \\
\hline 33 & MYPE2390 & MYPE2400 & 53 & $\rightarrow \rightarrow$ \\
\hline 34 & MYPE2470 & MYPE2480 & 20 & $\rightarrow \rightarrow$ \\
\hline
\end{tabular}


Angamuthu and Piramanayagam, IJPSR, 2013; Vol. 4(9): 3504-3517. $\quad$ E-ISSN: 0975-8232; P-ISSN: 2320-5148

\begin{tabular}{|c|c|c|c|c|}
\hline 35 & ruvB & ruvA & 35 & $\leftarrow \leftarrow$ \\
\hline 36 & MYPE2830 & MYPE2840 & 4 & $\rightarrow \leftarrow$ \\
\hline 37 & hprK & lgt & 14 & $\rightarrow \rightarrow$ \\
\hline 38 & MYPE3040 & rpe & 4 & $\rightarrow \leftarrow$ \\
\hline 39 & MYPE3070 & MYPE3080 & 62 & $\rightarrow \rightarrow$ \\
\hline 40 & mraW & MYPE3230 & 1 & $\rightarrow \rightarrow$ \\
\hline 41 & MYPE3350 & dgkA & 8 & $\rightarrow \rightarrow$ \\
\hline 42 & Era & MYPE3380 & 1 & $\rightarrow \rightarrow$ \\
\hline 43 & glyS & dnaG & 11 & $\rightarrow \rightarrow$ \\
\hline 44 & plsX & $\mathrm{rnc}$ & 26 & $\rightarrow \rightarrow$ \\
\hline 45 & tpiA & pgm & 11 & $\rightarrow \rightarrow$ \\
\hline 46 & MYPE4190 & MYPE4200 & 55 & $\rightarrow \leftarrow$ \\
\hline 47 & ulaA & tktA & 1 & $\rightarrow \rightarrow$ \\
\hline 48 & tktA & MYPE4520 & 14 & $\rightarrow \rightarrow$ \\
\hline 49 & MYPE4520 & MYPE4530 & 20 & $\rightarrow \rightarrow$ \\
\hline 50 & MYPE4600 & MYPE4610 & 1 & $\rightarrow \rightarrow$ \\
\hline 51 & MYPE4640 & MYPE4650 & 8 & $\rightarrow \rightarrow$ \\
\hline 52 & MYPE4730 & MYPE4740 & 17 & $\rightarrow \rightarrow$ \\
\hline 53 & MYPE4850 & MYPE4860 & 16 & $\rightarrow \rightarrow$ \\
\hline 54 & MYPE4930 & MYPE4940 & 20 & $\leftarrow \leftarrow$ \\
\hline 55 & hemN & miaA & 14 & $\leftarrow \leftarrow$ \\
\hline 56 & pdhA & pdhB & 1 & $\rightarrow \rightarrow$ \\
\hline 57 & oppC & орpB & 8 & $\leftarrow \leftarrow$ \\
\hline 58 & MYPE5580 & MYPE5590 & 8 & $\leftarrow \leftarrow$ \\
\hline 59 & MYPE5590 & MYPE5600 & 29 & $\leftarrow \leftarrow$ \\
\hline 60 & MYPE5620 & MYPE5630 & 14 & $\leftarrow \leftarrow$ \\
\hline 61 & MYPE5630 & gmk & 1 & $\leftarrow \leftarrow$ \\
\hline 62 & MYPE5680 & MYPE5690 & 17 & $\leftarrow \leftarrow$ \\
\hline 63 & glpQ & MYPE5760 & 8 & $\leftarrow \leftarrow$ \\
\hline 64 & Htp & MYPE5820 & 1 & $\leftarrow \leftarrow$ \\
\hline 65 & MYPE5870 & MYPE5880 & 1 & $\leftarrow \leftarrow$ \\
\hline 66 & MYPE5880 & MYPE5890 & 1 & $\leftarrow \leftarrow$ \\
\hline
\end{tabular}


Angamuthu and Piramanayagam, IJPSR, 2013; Vol. 4(9): 3504-3517. $\quad$ E-ISSN: 0975-8232; P-ISSN: 2320-5148

\begin{tabular}{|c|c|c|c|c|}
\hline 67 & MYPE5890 & MYPE5900 & 35 & $\leftarrow \leftarrow$ \\
\hline 68 & MYPE6010 & MYPE6020 & 17 & $\leftarrow \leftarrow$ \\
\hline 69 & MYPE6320 & MYPE6330 & 17 & $\leftarrow \leftarrow$ \\
\hline 70 & MYPE6440 & MYPE6450 & 20 & $\leftarrow \leftarrow$ \\
\hline 71 & MYPE6495 & MYPE6500 & 35 & $\leftarrow \leftarrow$ \\
\hline 72 & MYPE6600 & MYPE6610 & 11 & $\leftarrow \leftarrow$ \\
\hline 73 & MYPE6610 & MYPE6620 & 8 & $\leftarrow \leftarrow$ \\
\hline 74 & dhfR & thyA & 1 & $\leftarrow \leftarrow$ \\
\hline 75 & thy A & folD & 14 & $\leftarrow \leftarrow$ \\
\hline 76 & $\operatorname{araD}$ & sgaU & 20 & $\leftarrow \leftarrow$ \\
\hline 77 & sgaU & ulaD & 26 & $\leftarrow \leftarrow$ \\
\hline 78 & MYPE7375 & MYPE7380 & 53 & $\leftarrow \leftarrow$ \\
\hline 79 & nifU & nifS & 14 & $\leftarrow \leftarrow$ \\
\hline 80 & MYPE7570 & MYPE7580 & 38 & $\leftarrow \leftarrow$ \\
\hline 81 & MYPE7680 & MYPE7690 & 16 & $\leftarrow \leftarrow$ \\
\hline 82 & fruK & fruA & 47 & $\leftarrow \leftarrow$ \\
\hline 83 & fruA & MYPE7770 & 11 & $\leftarrow \leftarrow$ \\
\hline 84 & pyrB & pyrR & 1 & $\leftarrow \leftarrow$ \\
\hline 85 & gidB & MYPE8000 & 14 & $\rightarrow \longrightarrow$ \\
\hline 86 & MYPE8090 & MYPE8100 & 8 & $\leftarrow \leftarrow$ \\
\hline 87 & hsdR & hsdR & 50 & $\leftarrow \leftarrow$ \\
\hline 88 & MYPE8350 & $\operatorname{sig} \mathrm{A}$ & 20 & $\leftarrow \leftarrow$ \\
\hline 89 & MYPE8510 & MYPE8520 & 16 & $\leftarrow \leftarrow$ \\
\hline 90 & MYPE8530 & alaS & 17 & $\leftarrow \leftarrow$ \\
\hline 91 & $\operatorname{pot} \mathrm{A}$ & potB & 32 & $\rightarrow \rightarrow$ \\
\hline 92 & potB & potC & 8 & $\rightarrow \rightarrow$ \\
\hline 93 & potC & MYPE8600 & 13 & $\rightarrow \rightarrow$ \\
\hline 94 & MYPE8600 & gcp & 23 & $\rightarrow \rightarrow$ \\
\hline 95 & MYPE8620 & MYPE8630 & 17 & $\rightarrow \rightarrow$ \\
\hline 96 & MYPE8730 & oppF & 8 & $\leftarrow \leftarrow$ \\
\hline 97 & MYPE9020 & cysS & 8 & $\leftarrow \leftarrow$ \\
\hline 98 & truB & MYPE9290 & 4 & $\rightarrow \leftarrow$ \\
\hline
\end{tabular}




\begin{tabular}{lcccc}
\hline $\mathbf{9 9}$ & metS & MYPE9390 & 8 & $\leftarrow \leftarrow$ \\
$\mathbf{1 0 0}$ & MYPE9420 & MYPE9430 & 4 & $\rightarrow \leftarrow$ \\
$\mathbf{1 0 1}$ & MYPE9440 & MYPE9450 & 13 & $\leftarrow \leftarrow$ \\
$\mathbf{1 0 2}$ & MYPE9640 & MYPE9650 & 11 & $\leftarrow \leftarrow$ \\
$\mathbf{1 0 3}$ & cbiO & cbiO & 16 & $\leftarrow \leftarrow$ \\
$\mathbf{1 0 4}$ & MYPE9870 & MYPE9880 & 8 & $\leftarrow$ \\
$\mathbf{1 0 5}$ & MYPE9880 & MYPE9890 & 28 & $\leftarrow$ \\
$\mathbf{1 0 6}$ & rpmC & rplP & 14 & $\leftarrow$ \\
$\mathbf{1 0 7}$ & rplW & rplD & 1 & $\leftarrow$ \\
$\mathbf{1 0 8}$ & rplD & rplC & 1 & $\leftarrow$ \\
$\mathbf{1 0 9}$ & MYPE10380 & ksgA & 20 & \\
\hline
\end{tabular}

TABLE 4: OVERLAPPING GENE PAIRS COMMON TO BOTH M. HOMINIS AND M. PENETRANS

\begin{tabular}{|c|c|c|c|c|c|c|c|c|}
\hline \multirow{2}{*}{ S. No. } & \multirow{2}{*}{$\begin{array}{l}\text { Gene } \\
\text { Name }\end{array}$} & \multirow{2}{*}{ Function } & \multicolumn{2}{|c|}{ Locus } & \multicolumn{2}{|c|}{$\begin{array}{l}\text { Length of } \\
\text { Overlap }\end{array}$} & \multicolumn{2}{|c|}{$\begin{array}{l}\text { Direction of } \\
\text { Overlap }\end{array}$} \\
\hline & & & M.hom & M.pen & M.hom & M.pen & M.hom & M.pen \\
\hline 1 & $\begin{array}{l}\text { prfA: } \\
\text { hemK }\end{array}$ & $\begin{array}{l}\text { peptide chain release factor } 1: \\
\text { S-adenosylmethionine-dependent } \\
\text { methyltransferase }\end{array}$ & $\begin{array}{l}\text { MHO_1600: } \\
\text { MHO_1610 }\end{array}$ & $\begin{array}{l}\text { MYPE650: } \\
\text { MYPE660 }\end{array}$ & 1 & 35 & $\rightarrow$ & $\rightarrow$ \\
\hline 2 & $\begin{array}{l}\text { infB: } \\
\text { rbfA }\end{array}$ & $\begin{array}{l}\text { translation initiation factor IF-2: } \\
\text { ribosome-binding factor A }\end{array}$ & $\begin{array}{l}\text { MHO_5270: } \\
\text { MHO_5280 }\end{array}$ & $\begin{array}{l}\text { MYPE1110: } \\
\text { MYPE1120 }\end{array}$ & 14 & 17 & $\begin{array}{l}\rightarrow \\
\rightarrow\end{array}$ & $\begin{array}{l}\rightarrow \\
\rightarrow\end{array}$ \\
\hline 3 & $\begin{array}{l}\text { gatA: } \\
\text { gatB }\end{array}$ & $\begin{array}{c}\text { aspartyl/glutamyl-tRNA amidotransferase } \\
\text { subunit A: aspartyl/glutamyl-tRNA } \\
\text { amidotransferase subunit B }\end{array}$ & $\begin{array}{l}\text { MHO_0440: } \\
\text { MHO_0450 }\end{array}$ & $\begin{array}{l}\text { MYPE2100: } \\
\text { MYPE2110 }\end{array}$ & 8 & 23 & $\rightarrow$ & $\rightarrow$ \\
\hline 4 & $\begin{array}{l}\text { ruvB: } \\
\text { ruvA }\end{array}$ & $\begin{array}{l}\text { Holliday junction DNA helicase B: } \\
\text { holliday junction DNA helicase }\end{array}$ & $\begin{array}{l}\text { MHO_1850: } \\
\text { MHO_1860 }\end{array}$ & $\begin{array}{l}\text { MYPE2780: } \\
\text { MYPE2790 }\end{array}$ & 26 & 35 & $\rightarrow$ & $\leftarrow$ \\
\hline 5 & $\begin{array}{l}\text { plsX: } \\
\text { rnc }\end{array}$ & $\begin{array}{l}\text { putative glycerol-3-phosphate } \\
\text { acyltransferase PlsX: ribonuclease III }\end{array}$ & $\begin{array}{l}\text { MHO_4680: } \\
\text { MHO_4690 }\end{array}$ & $\begin{array}{l}\text { MYPE3640: } \\
\text { MYPE3650 }\end{array}$ & 11 & 26 & $\rightarrow$ & $\rightarrow$ \\
\hline 6 & $\begin{array}{l}\text { oppC: } \\
\text { oppB }\end{array}$ & $\begin{array}{l}\text { oligopeptide transport system permease } \\
\text { protein oligopeptide transport system } \\
\text { permease protein }\end{array}$ & $\begin{array}{l}\text { MHO_1740: } \\
\text { MHO_1750 }\end{array}$ & $\begin{array}{l}\text { MYPE5540: } \\
\text { MYPE5550 }\end{array}$ & 17 & 8 & $\begin{array}{l}\rightarrow \\
\rightarrow\end{array}$ & $\leftarrow$ \\
\hline 7 & $\begin{array}{l}\text { nifU: } \\
\text { nifS }\end{array}$ & $\begin{array}{l}\text { nitrogen fixation protein NifU: } \\
\text { aminotransferase NifS }\end{array}$ & $\begin{array}{l}\text { MHO_1230: } \\
\text { MHO_1240 }\end{array}$ & $\begin{array}{l}\text { MYPE7420: } \\
\text { MYPE7430 }\end{array}$ & 14 & 14 & $\vec{\rightarrow}$ & $\leftarrow$ \\
\hline 8 & $\begin{array}{l}\text { PotA: } \\
\text { potB }\end{array}$ & $\begin{array}{l}\text { spermidine/putrescine transport ATP- } \\
\text { binding protein: spermidine/putrescine } \\
\text { transport system permease }\end{array}$ & $\begin{array}{l}\text { MHO_4450: } \\
\text { MHO_4460 }\end{array}$ & $\begin{array}{l}\text { MYPE8570: } \\
\text { MYPE8580 }\end{array}$ & 32 & 32 & $\vec{\rightarrow}$ & $\begin{array}{l}\rightarrow \\
\rightarrow\end{array}$ \\
\hline 9 & $\begin{array}{l}\text { potB: } \\
\text { potC }\end{array}$ & $\begin{array}{l}\text { spermidine/putrescine transport system } \\
\text { permease: spermidine/putrescine transport } \\
\text { system permease Po }\end{array}$ & $\begin{array}{l}\text { MHO_4460: } \\
\text { MHO_4470 }\end{array}$ & $\begin{array}{l}\text { MYPE8580: } \\
\text { MYPE8590 }\end{array}$ & 8 & 8 & $\begin{array}{l}\rightarrow \\
\rightarrow\end{array}$ & $\begin{array}{l}\rightarrow \\
\rightarrow\end{array}$ \\
\hline
\end{tabular}


10 cbiO: cobalt transporter ATP-binding subunit: cbiO cobalt transporter ATP-binding subunit $\begin{array}{ll}\text { MHO_1970: } & \text { MYPE9760: } \\ \text { MHO_1980 } & \text { MYPE9770 }\end{array}$

12 rplD: $\begin{array}{ll}\text { rplW: } & \text { 50S ribosomal proteinL23 } \\ \text { rplD } & 50 \text { S ribosomal protein } \mathrm{L4}\end{array}$
50S ribosomal protein L4: $50 \mathrm{~S}$ ribosomal protein L3 $\begin{array}{ll}\text { rplW: } & \text { 50S ribosomal proteinL23: } \\ \text { rplD } & 50 \text { S ribosomal protein L4 }\end{array}$

rplC
MHO_2960: MHO_2970

10

16

MYPE1010:

MYPE1010

MHO_2970:

MHO_2980
MYPE1017

0 : MYPE1018

TABLE 5: GENE PAIRS THAT OVERLAP IN $M$. HOMINIS BUT ARE SPLIT IN M. PENETRANS

\begin{tabular}{|c|c|c|c|c|c|}
\hline S. No. & $\begin{array}{l}\text { Gene } \\
\text { name }\end{array}$ & Function & Locus & Length & Direction \\
\hline 1 & $\begin{array}{l}\text { atpA: } \\
\text { atpG }\end{array}$ & $\begin{array}{l}\text { ATP synthase subunit alpha: } \\
\text { ATP synthase subunit gamma }\end{array}$ & $\begin{array}{l}\text { MHO_0240: } \\
\text { MHO_0250 }\end{array}$ & 22 & $\rightarrow$ \\
\hline 2 & $\begin{array}{l}\text { atpD: } \\
\text { atpC }\end{array}$ & $\begin{array}{l}\text { ATP synthase subunit beta: } \\
\text { ATP synthase subunit epsilon }\end{array}$ & $\begin{array}{l}\text { MHO_0260: } \\
\text { MHO_0270 }\end{array}$ & 1 & $\rightarrow$ \\
\hline 3 & $\begin{array}{l}\text { scpA: } \\
\text { scpB }\end{array}$ & $\begin{array}{c}\text { segregation and condensation protein A: segregation } \\
\text { and condensation protein B }\end{array}$ & $\begin{array}{l}\text { MHO_0400: } \\
\text { MHO_0410 }\end{array}$ & 11 & $\rightarrow$ \\
\hline 4 & $\begin{array}{l}\text { scpB: } \\
\text { rluC }\end{array}$ & $\begin{array}{l}\text { segregation and condensation protein B: ribosomal } \\
\text { large subunit pseudouridine synthase }\end{array}$ & $\begin{array}{l}\text { MHO_0410: } \\
\text { MHO_0420 }\end{array}$ & 14 & $\rightarrow$ \\
\hline 5 & $\begin{array}{l}\text { gatC: } \\
\text { gatA }\end{array}$ & $\begin{array}{l}\text { glutamyl- tRNA(gln)amidotransferase subunit C: } \\
\text { glutamyl-tRNA(gln)amidotransferase subunit A }\end{array}$ & $\begin{array}{l}\text { MHO_0420: } \\
\text { MHO_0440 }\end{array}$ & 11 & $\rightarrow$ \\
\hline 6 & $\begin{array}{l}\text { thrS: } \\
\text { trpS }\end{array}$ & $\begin{array}{c}\text { threonyl-tRNA synthetase: Tryptophanyl-tRNA } \\
\text { synthetase }\end{array}$ & $\begin{array}{l}\text { MHO_1050: } \\
\text { MHO_1060 }\end{array}$ & 1 & $\leftarrow$ \\
\hline 7 & $\begin{array}{l}\text { nusB: } \\
\text { tlyA }\end{array}$ & $\begin{array}{l}\text { N utilization NusB-like protein: } \\
\text { Hemolysin A }\end{array}$ & $\begin{array}{l}\text { MHO_1210: } \\
\text { MHO_1220 }\end{array}$ & 17 & $\rightarrow$ \\
\hline 8 & $\begin{array}{l}\text { tlyA: } \\
\text { nifS }\end{array}$ & $\begin{array}{c}\text { Hemolysin A: Nitrogen fixation protein } \\
\text { NifS(aminotransferase }\end{array}$ & $\begin{array}{l}\text { MHO_1220: } \\
\text { MHO_1230 }\end{array}$ & 1 & $\rightarrow$ \\
\hline 9 & $\begin{array}{l}\text { oppD: } \\
\text { oppF }\end{array}$ & $\begin{array}{l}\text { putative oligopeptide transport ATP-binding protein: } \\
\text { putative oligopeptide transport ATP-binding protein }\end{array}$ & $\begin{array}{l}\text { MHO_1540: } \\
\text { MHO_1550 }\end{array}$ & 8 & $\rightarrow$ \\
\hline 10 & $\begin{array}{l}\text { Cmk: } \\
\text { engA }\end{array}$ & $\begin{array}{l}\text { Cytidylate kinase: } \\
\text { GTP-binding protein engA }\end{array}$ & $\begin{array}{l}\text { MHO_1670: } \\
\text { MHO_1680 }\end{array}$ & 14 & $\rightarrow$ \\
\hline 11 & $\begin{array}{l}\text { hisS: } \\
\text { aspS }\end{array}$ & $\begin{array}{l}\text { Histidyl-tRNA synthetase: } \\
\text { Aspartyl-tRNA synthetase }\end{array}$ & $\begin{array}{l}\text { MHO_1870: } \\
\text { MHO_1880 }\end{array}$ & 17 & $\rightarrow$ \\
\hline 12 & $\begin{array}{l}\text { Dgk: } \\
\text { hpt }\end{array}$ & $\begin{array}{l}\text { Deoxyguanosine kinase: } \\
\text { Hypoxanthine-guanine phosphoribosyltransferase } \\
\text { (HGP... }\end{array}$ & $\begin{array}{l}\text { MHO_1930: } \\
\text { MHO_1940 }\end{array}$ & 18 & $\rightarrow$ \\
\hline 13 & $\begin{array}{l}\text { Smf: } \\
\text { nfo }\end{array}$ & $\begin{array}{l}\text { DNA processing protein smf: } \\
\text { Endonuclease IV }\end{array}$ & $\begin{array}{l}\text { MHO_2130: } \\
\text { MHO_2140 }\end{array}$ & 9 & 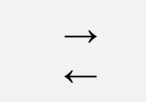 \\
\hline 14 & $\begin{array}{l}\text { infA: } \\
\text { map }\end{array}$ & $\begin{array}{l}\text { translation initiation factor IF-1: methionine } \\
\text { aminopeptidase (MAP) }\end{array}$ & $\begin{array}{l}\text { MHO_2760: } \\
\text { MHO_2770 }\end{array}$ & 1 & $\leftarrow$ \\
\hline 15 & $\begin{array}{l}\text { Map: } \\
\text { adk }\end{array}$ & $\begin{array}{l}\text { methionine aminopeptidase (MAP): Adenylate kinase } \\
\text { (ATP-AMP transphosphorylase) }\end{array}$ & $\begin{array}{l}\text { MHO_2770: } \\
\text { MHO_2780 }\end{array}$ & 10 & $\leftarrow$ \\
\hline 16 & $\begin{array}{l}\text { Adk: } \\
\text { secY }\end{array}$ & $\begin{array}{c}\text { Adenylate kinase (ATP-AMP transphosphorylase): } \\
\text { Preprotein translocase secY subunit }\end{array}$ & $\begin{array}{l}\text { MHO_2780: } \\
\text { MHO_2790 }\end{array}$ & 7 & $\leftarrow$ \\
\hline
\end{tabular}




\begin{tabular}{|c|c|c|c|c|c|}
\hline 17 & $\begin{array}{l}\text { secY: } \\
\text { rplO }\end{array}$ & $\begin{array}{l}\text { Preprotein translocase secY subunit: } \\
\text { 50S ribosomal protein L15 }\end{array}$ & $\begin{array}{l}\text { MHO_2790: } \\
\text { MHO_2800 }\end{array}$ & 1 & $\leftarrow$ \\
\hline 18 & $\begin{array}{l}\text { rplN: } \\
\text { rpsQ }\end{array}$ & $\begin{array}{l}\text { 50S ribosomal protein } \mathrm{L} 14 \text { : } \\
\text { 30S ribosomal protein } \mathrm{S} 17\end{array}$ & $\begin{array}{l}\text { MHO_2880: } \\
\text { MHO_2890 }\end{array}$ & 8 & $\leftarrow$ \\
\hline 19 & $\begin{array}{l}\text { rpsQ: } \\
\text { rpmC }\end{array}$ & $\begin{array}{l}\text { 30S ribosomal protein S17: } \\
50 \text { S ribosomal protein L29 }\end{array}$ & $\begin{array}{l}\text { MHO_2890: } \\
\text { MHO_2900 }\end{array}$ & 1 & $\leftarrow$ \\
\hline 20 & $\begin{array}{l}\text { rplP: } \\
\text { rpsC }\end{array}$ & $\begin{array}{l}\text { 50S ribosomal protein L16: } \\
\text { 30S ribosomal protein S3 }\end{array}$ & $\begin{array}{l}\text { MHO_2910: } \\
\text { MHO_2920 }\end{array}$ & 23 & $\leftarrow$ \\
\hline 21 & $\begin{array}{l}\text { rpsC: } \\
\text { rplV }\end{array}$ & $\begin{array}{l}\text { 30S ribosomal protein } \mathrm{S} 3 \text { : } \\
50 \mathrm{~S} \text { ribosomal protein } \mathrm{L} 22\end{array}$ & $\begin{array}{l}\text { MHO_2920: } \\
\text { MHO_2930 }\end{array}$ & 1 & $\leftarrow$ \\
\hline 22 & $\begin{array}{l}\text { rpsS: } \\
\text { rplB }\end{array}$ & $\begin{array}{l}\text { 30S ribosomal protein } \mathrm{S} 19 \text { : } \\
50 \mathrm{~S} \text { ribosomal protein } \mathrm{L} 2\end{array}$ & $\begin{array}{l}\text { MHO_2940: } \\
\text { MHO_2950 }\end{array}$ & 1 & $\leftarrow$ \\
\hline 23 & $\begin{array}{l}\text { lspA: } \\
\text { ileS }\end{array}$ & $\begin{array}{l}\text { lipoprotein signal peptidase: } \\
\text { Isoleucyl-tRNA synthetase }\end{array}$ & $\begin{array}{l}\text { MHO_3040: } \\
\text { MHO_3050 }\end{array}$ & 11 & $\leftarrow$ \\
\hline 24 & $\begin{array}{l}\text { atpD: } \\
\text { atpA }\end{array}$ & $\begin{array}{l}\text { ATP synthase subunit beta: } \\
\text { ATP synthase subunit alpha }\end{array}$ & $\begin{array}{l}\text { MHO_3120: } \\
\text { MHO_3130 }\end{array}$ & 1 & $\leftarrow$ \\
\hline 25 & $\begin{array}{l}\text { hsdS: } \\
\text { hsdS }\end{array}$ & $\begin{array}{l}\text { Type I restriction enzyme specificity protein: } \\
\text { Type I restriction enzyme specificity protein }\end{array}$ & $\begin{array}{l}\text { MHO_3220: } \\
\text { MHO_3230 }\end{array}$ & 23 & $\leftarrow$ \\
\hline 26 & $\begin{array}{l}\text { gidB: } \\
\text { prs }\end{array}$ & $\begin{array}{l}\text { methyltransferase gidB: } \\
\text { ribose-phosphate pyrophosphokinase }\end{array}$ & $\begin{array}{l}\text { MHO_3540: } \\
\text { MHO_3550 }\end{array}$ & 26 & $\leftarrow$ \\
\hline 27 & $\begin{array}{l}\text { Ldh: } \\
\text { pgi }\end{array}$ & $\begin{array}{l}\text { L-lactate dehydrogenase: } \\
\text { glucose-6-phosphate isomerase }\end{array}$ & $\begin{array}{l}\text { MHO_3580: } \\
\text { MHO_3590 }\end{array}$ & 8 & $\leftarrow$ \\
\hline 28 & $\begin{array}{l}\text { Pgi: } \\
\text { rpsA }\end{array}$ & $\begin{array}{l}\text { glucose-6-phosphate isomerase: } \\
\text { 30S ribosomal protein } \mathrm{S} 1\end{array}$ & $\begin{array}{l}\text { MHO_3590: } \\
\text { MHO_3600 }\end{array}$ & 11 & $\leftarrow$ \\
\hline 29 & $\begin{array}{l}\text { pepF: } \\
\text { apt }\end{array}$ & $\begin{array}{l}\text { Oligoendopeptidase F: } \\
\text { Adenine phosphoribosyl transferase }\end{array}$ & $\begin{array}{l}\text { MHO_3670: } \\
\text { MHO_3680 }\end{array}$ & 1 & $\leftarrow$ \\
\hline 30 & $\begin{array}{l}\operatorname{md} 2: \\
\operatorname{md} 1\end{array}$ & $\begin{array}{l}\text { ABC transporter ATP binding protein: } \\
\text { ABC transporter ATP binding protein }\end{array}$ & $\begin{array}{l}\text { MHO_3820: } \\
\text { MHO_3830 }\end{array}$ & 8 & $\leftarrow$ \\
\hline 31 & $\begin{array}{l}\text { engB: } \\
\text { gcp }\end{array}$ & $\begin{array}{l}\text { putative GTP-binding protein engB: } \\
\text { O-sialoglycoprotein endopeptidase }\end{array}$ & $\begin{array}{l}\text { MHO_3940: } \\
\text { MHO_3950 }\end{array}$ & 1 & $\leftarrow$ \\
\hline 32 & $\begin{array}{l}\text { DnaE: } \\
\text { polA }\end{array}$ & $\begin{array}{l}\text { DNA polymerase III subunit alpha: } \\
\text { DNA polymerase I }\end{array}$ & $\begin{array}{l}\text { MHO_4120: } \\
\text { MHO_4130 }\end{array}$ & 14 & $\rightarrow$ \\
\hline 33 & $\begin{array}{l}\text { recD: } \\
\text { pth }\end{array}$ & $\begin{array}{c}\text { Exodeoxyribonuclease V subunit alpha: Peptidyl- } \\
\text { tRNA hydrolase }\end{array}$ & $\begin{array}{l}\text { MHO_4200: } \\
\text { MHO_4210 }\end{array}$ & 1 & $\rightarrow$ \\
\hline 34 & $\begin{array}{l}\text { Pth: } \\
\text { tilS }\end{array}$ & $\begin{array}{l}\text { Peptidyl-tRNA hydrolase: } \\
\text { tRNA(Ile)-lysidine synthase }\end{array}$ & $\begin{array}{l}\text { MHO_4210: } \\
\text { MHO_4220 }\end{array}$ & 14 & $\rightarrow$ \\
\hline 35 & $\begin{array}{l}\text { pyrH: } \\
\text { frr }\end{array}$ & $\begin{array}{l}\text { Uridylate kinase smbA: } \\
\text { ribosome recycling factor }\end{array}$ & $\begin{array}{l}\text { MHO_4600: } \\
\text { MHO_4610 }\end{array}$ & 1 & $\rightarrow$ \\
\hline 36 & $\begin{array}{l}\text { infB: } \\
\text { rbfA }\end{array}$ & $\begin{array}{l}\text { translation initiation factor IF-2: } \\
\text { ribosome-binding factor A }\end{array}$ & $\begin{array}{l}\text { MHO_5270: } \\
\text { MHO_5280 }\end{array}$ & 14 & $\vec{\rightarrow}$ \\
\hline
\end{tabular}


TABLE 6: GENE PAIRS THAT OVERLAP IN M. PENETRANS BUT ARE SPLIT IN M. HOMINIS

\begin{tabular}{|c|c|c|c|c|c|}
\hline S. No. & $\begin{array}{l}\text { Gene } \\
\text { name }\end{array}$ & Function & Locus & Length & Direction \\
\hline 1 & $\begin{array}{l}\text { nfo: } \\
\text { fur }\end{array}$ & $\begin{array}{l}\text { endonuclease IV: } \\
\text { ferric uptake regulation protein }\end{array}$ & $\begin{array}{l}\text { MYPE1190: } \\
\text { MYPE1200 }\end{array}$ & 13 & $\rightarrow$ \\
\hline 2 & $\begin{array}{c}\text { Dam: } \\
\text { dam }\end{array}$ & $\begin{array}{l}\text { adenine-specific DNA methyltransferase: } \\
\text { adenine-specific DNA methyltransferase }\end{array}$ & $\begin{array}{l}\text { MYPE1780: } \\
\text { MYPE1790 }\end{array}$ & 38 & $\rightarrow$ \\
\hline 3 & $\begin{array}{l}\text { engA: } \\
\text { gpsA }\end{array}$ & $\begin{array}{c}\text { GTP-binding protein EngA: } \\
\text { NAD-dependent glycerol-3-phosphate } \\
\text { dehydrogenase }\end{array}$ & $\begin{array}{l}\text { MYPE2290: } \\
\text { MYPE2300 }\end{array}$ & 11 & $\rightarrow$ \\
\hline 4 & $\begin{array}{l}\text { hprK: } \\
\text { lgt }\end{array}$ & $\begin{array}{l}\text { HPr kinase/phosphorylase: prolipoprotein } \\
\text { diacylglyceryl transferase }\end{array}$ & $\begin{array}{l}\text { MYPE2990: } \\
\text { MYPE3000 }\end{array}$ & 14 & $\rightarrow$ \\
\hline 5 & $\begin{array}{l}\text { glyS: } \\
\text { dnaG }\end{array}$ & $\begin{array}{l}\text { glycyl-tRNA synthetase: } \\
\text { DNA primase }\end{array}$ & $\begin{array}{l}\text { MYPE3390: } \\
\text { MYPE3400 }\end{array}$ & 11 & $\rightarrow$ \\
\hline 6 & $\begin{array}{l}\text { tpiA: } \\
\text { pgm }\end{array}$ & $\begin{array}{l}\text { triose phosphate isomerase [Mycoplasma } \\
\text { penetrans H: phosphoglyceromutase }\end{array}$ & $\begin{array}{l}\text { MYPE3730: } \\
\text { MYPE3740 }\end{array}$ & 11 & $\rightarrow$ \\
\hline 7 & $\begin{array}{l}\text { ulaA: } \\
\text { tktA }\end{array}$ & $\begin{array}{l}\text { ascorbate-specific PTS system enzyme IIC: } \\
\text { transketolase }\end{array}$ & $\begin{array}{l}\text { MYPE4500: } \\
\text { MYPE4510 }\end{array}$ & 1 & $\rightarrow$ \\
\hline 8 & $\begin{array}{l}\text { hemN: } \\
\text { miaA }\end{array}$ & $\begin{array}{l}\text { coproporphyrinogen III oxidase : } \\
\text { tRNA-isopentenyl pyrophosphate transferase }\end{array}$ & $\begin{array}{l}\text { MYPE5010: } \\
\text { MYPE5020 }\end{array}$ & 14 & $\leftarrow$ \\
\hline 9 & $\begin{array}{l}\text { pdhA: } \\
\text { pdhB }\end{array}$ & $\begin{array}{l}\text { Pyruvate dehydrogenase E1 component subunit } \\
\text { alpha: pyruvate dehydrogenase E1 component } \\
\text { subunit beta }\end{array}$ & $\begin{array}{l}\text { MYPE5080: } \\
\text { MYPE5090 }\end{array}$ & 1 & $\rightarrow$ \\
\hline 10 & $\begin{array}{l}\text { dhfR: } \\
\text { thyA }\end{array}$ & $\begin{array}{l}\text { dihyrofolatereductase: } \\
\text { thymidylate synthase }\end{array}$ & $\begin{array}{l}\text { MYPE6860: } \\
\text { MYPE6870 }\end{array}$ & 1 & $\leftarrow$ \\
\hline 11 & $\begin{array}{l}\text { thyA: } \\
\text { folD }\end{array}$ & $\begin{array}{l}\text { thymidylate synthase: } \\
\text { methylenetetrahydrofolate dehydrogenase }\end{array}$ & $\begin{array}{l}\text { MYPE6870: } \\
\text { MYPE6880 }\end{array}$ & 14 & $\leftarrow$ \\
\hline 12 & $\begin{array}{l}\text { araD: } \\
\text { sgaU }\end{array}$ & $\begin{array}{l}\text { L-ribulose-5-phosphate 4-epimerase: } \\
\text { L-xylulose 5-phosphate } 3 \text {-epimerase }\end{array}$ & $\begin{array}{l}\text { MYPE7160: } \\
\text { MYPE7170 }\end{array}$ & 20 & $\leftarrow$ \\
\hline 13 & $\begin{array}{l}\text { sgaU: } \\
\text { ulaD }\end{array}$ & $\begin{array}{l}\text { L-xylulose 5-phosphate 3-epimerase: } \\
\text { 3-keto-L-gulonate-6-phosphate decarboxylase }\end{array}$ & $\begin{array}{l}\text { MYPE7170: } \\
\text { MYPE7180 }\end{array}$ & 26 & $\leftarrow$ \\
\hline 14 & $\begin{array}{l}\text { fruK: } \\
\text { fruA }\end{array}$ & $\begin{array}{l}\text { 1-phosphofructokinase: PTS system fructose- } \\
\text { specific IIABC component }\end{array}$ & $\begin{array}{l}\text { MYPE7750: } \\
\text { MYPE7760 }\end{array}$ & 47 & $\leftarrow$ \\
\hline 15 & $\begin{array}{l}\text { pyrB: } \\
\text { pyrR }\end{array}$ & $\begin{array}{l}\text { aspartate carbamoyltransferase catalytic subunit: } \\
\text { pyrimidine regulatory protein PyrR }\end{array}$ & $\begin{array}{l}\text { MYPE7890: } \\
\text { MYPE7900 }\end{array}$ & 1 & $\leftarrow$ \\
\hline 16 & $\begin{array}{l}\text { hsdR: } \\
\text { hsdR }\end{array}$ & $\begin{array}{l}\text { type I restriction-modification system R subunit: } \\
\text { type I restriction-modification system R subunit. }\end{array}$ & $\begin{array}{l}\text { MYPE8220: } \\
\text { MYPE8230 }\end{array}$ & 50 & $\leftarrow$ \\
\hline 17 & $\begin{array}{l}\text { rpmC: } \\
\text { rplP }\end{array}$ & $\begin{array}{l}\text { ribosomal protein L29: } \\
\text { 50S ribosomal protein L16 }\end{array}$ & $\begin{array}{l}\text { MYPE10100: } \\
\text { MYPE10110 }\end{array}$ & 14 & $\leftarrow$ \\
\hline
\end{tabular}

CONCLUSION: Computational comparative genomic analysis of $M$. hominis and $M$. penetrans has provided a breakthrough towards the understanding of the evolution of these genomes. It has been analyzed that there is no correlation between the genome size, number of genes and number of overlapping gene pairs. Most of the overlapping genes in $M$. hominis and $M$. penetrans are formed due to loss of a stop codon or frame shift.
The current study reveals the significance of overlapping genes in bringing about the genome compaction of Mycoplasmas during the course of reductive evolution.

\section{REFERENCES:}

1. Ho MR, Tsai KW and Lin WC: A unified framework of overlapping genes: towards the origination and endogenic regulation. Genomics 2012; 100(4):231-9. 
2. Kim DS, Cho CY, Huh JW, Kim HS and Cho HG EVOG: a database for evolutionary analysis of overlapping genes. Nucleic Acids Research 2009; 37: D698-702.

3. Clark MA, Baumann L, Thao ML, Moran NA, and Baumann P: Degenerative minimalism in the genome of a psyllid endosymbiont. Journal of Bacteriology 2001; 183: 1853-1861.

4. Fukuda Y, Washio T, and Tomita M: Comparative study of overlapping genes in the genomes of Mycoplasma genitalium and Mycoplasma pneumoniae. Nucleic Acids Research 1999; 27: 1847-1853.

5. Fukuda Y, Nakayama Y, and Tomita M: On dynamics of overlapping genes in bacterial genomes. Gene 2003; 323:181-187.

6. Sakharkar KR, Sakharkar MK, Verma C and Chow VT: Comparative study of overlapping genes in bacteria, with special reference to Rickettsia prowazekii and Rickettsia conorii. International Journal of Systematic and Evolutionary Microbiology 2005; 55:1205-1209.

7. Cock PJ and Whitworth DE: Evolution of relative reading frame bias in unidirectional prokaryotic gene overlaps. Molecular Biology and Evolution 2010; 27: 753-756.

8. Lillo F and Krakauer DC: A statistical analysis of the three- fold evolution of genomic compression through frame overlaps in prokaryotes. Biology Direct 2007; 2:22.

9. Keese PK and Gibbs A: Origins of genes: "big bang" or continuous creation? Proceedings of the National Academy of Sciences of the United States of America 1992; 89: 9489-9493.

10. Johnson ZI and Chisholm SW: Properties of overlapping genes are conserved across microbial genomes. Genome Research 2004; 14: 2268-2272.

11. Lipman DJ: Making (anti)sense of non-coding sequence conservation. Nucleic Acids Research 1997; 25: 35803583 .
12. Yelin R, Dahary D, Sorek R, et al: Widespread occurrence of antisense transcription in the human genome. Nature Biotechnology 2003; 21:379-386.

13. Luo Y, Fu C, Zhang DY and Lin K: Overlapping genes as rare genomic markers: the phylogeny of gammaProteobacteria as a case study. Trends in Genetics 2006; 22: 593-596.

14. Weisburg WG, Tully JG, Rose DL, Petzel JP, Oyaizu H, Yang D, Mandelco L, Sechrest J, Lawrence TG, Etten VJ, Maniloff $\mathrm{J}$ and Woese CR: A Phylogenetic analysis of mycoplasmas: basis for their classification. Journal of Bacteriology 1989; 171: 6455-6467.

15. Fadiel A, Eichenbaum KD, El Semary N, and Epperson B: Mycoplasma genomics: tailoring the genome for minimal life requirements through reductive evolution. Frontiers in Bioscience. 2007; 12:2020-8.

16. Razin S and Hayflick L: Highlights of mycoplasma research-An historical perspective. Biologicals 2010; 38: 183-190.

17. Sakharkar KR and Chow VT: Strategies for genome reduction in microbial genomes. Genome Informatics 2005; 16: 69-75.

18. Rogozin IB, Spiridonov AN, Sorokin AV, Wolf YI, Jordan IK, Tatusov RL, and Koonin EV: Purifying and directional selection in overlapping prokaryotic genes. Trends in Genetics 2002; 18: 228-232.

19. Eyre-Walker A: The distance between Escherichia coli genes is related to gene expression levels. Journal of Bacteriol 1995; 177: 5368-5369.

20. Krakauer DC: Stability and evolution of overlapping genes. International Journal of Systematic and Evolutionary Microbiology 2000; 54: 731-739.

\section{How to cite this article:}

Angamuthu K and Piramanayagam S: Comparative study of overlapping genes in the genomes of Mycoplasma hominis and Mycoplasma penetrans. Int J Pharm Sci Res 2013: 4(9); 3504-3517. doi: 10.13040/IJPSR. 0975-8232.4(9).3504-17

All @ 2013 are reserved by International Journal of Pharmaceutical Sciences and Research. This Journal licensed under a Creative Commons Attribution-NonCommercial-ShareAlike 3.0 Unported License.

This article can be downloaded to ANDROID OS based mobile. Scan QR Code using Code/Bar Scanner from your mobile. (Scanners are available on Google Playstore) 